\title{
Development of next-generation high-throughput MX beamline at SPring-8
}

\author{
Masaki Yamamoto ${ }^{1,3^{*}}$, Kunio Hirata ${ }^{1}$, Keitaro Yamashita ${ }^{1,2}$, Seiki Baba ${ }^{1,3}$, Kazuya \\ Hasegawa ${ }^{1,3}$, Naoki Sakai ${ }^{1}$, Yoshiaki Kawano ${ }^{1}$, Hironori Murakami ${ }^{1,3}$ and Takashi \\ Kumasaka ${ }^{1,3}$ \\ ${ }^{1}$ RIKEN SPring-8 Center, 1-1-1 Kouto, Sayo, Hyogo 679-5148, Japan, *yamamoto@riken.jp \\ 2 The University of Tokyo, 7-3-1 Hongo, Bunkyo-ku, Tokyo 113-0033, Japan \\ 3 Japan Synchrotron Radiation Research Institute (JASRI), 1-1-1 Kouto, Sayo, Hyogo 679- \\ 5198, Japan,
}

In the past decade, macromolecular crystallography (MX) remarkably developed by brilliant micro-focus beamlines, rapid-readout detectors and sample changers, and the progress has opened up protein micro crystallography. At the micro-focus beamline BL32XU at SPring-8, we have been developing the automated data collection system named ZOO [1, 2]. The system enables unattended data collection and dramatically shortens time for data collection. Data quality can be well controlled by automated crystal selection and radiation damage control implemented. The enhanced experimental efficiency and data quality accelerate the accumulation of better data sets within the limited beam time. Merging many data sets improves the signal-to-noise ratio and achieves higher resolution for structural analysis. These benefits of using ZOO system have accelerated the high-resolution structural analysis of challenging samples.

For crystals larger than $50 \mu \mathrm{m}$, ZOO system is capable of collecting a few hundred full data sets within a day. The capability benefits to fragment based drug design (FBDD) by examining a large number of crystals in complex with ligands. For this purpose, we are currently working on scrap and build process of the beamline BL45XU at SPring-8. SAXS activity is switched to a fully automated high-throughput MX beamline with ZOO. The expansion of developed technologies among SPring-8 beamlines eventually benefits to all users. Simultaneously with the beamline development, we are establishing an automatic structural analysis pipeline especially focused on developing sample mounting techniques continuous from crystallization for more efficient data collection.

We will present the current status and the future prospects of the next-generation highthroughput MX at SPring-8.

\section{References}

[1] Yamashita K., Hirata K., Yamamoto M. (2018) Acta Cryst. D74, 441-449

[2] Hirata, K., Yamashita, K., et al.and Yamamoto, M. (2019) Acta Cryst. D 75, 138-150 